\title{
SMALL BODIES IN THE OUTER SOLAR SYSTEM
}

\author{
BRIAN G. MARSDEN \\ Harvard-Smithsonian Center for Astrophysics \\ Cambridge, Massachusetts, U.S.A.
}

This report is a continuation of three earlier reviews (Marsden 1996a, 1996b, 1998) that included a summary of our orbital knowledge of the Kuiper Belt. Presented at conferences held in the middle of 1994, 1995 and 1996, respectively, these reviews showed the steadily developing picture of a system dominated by the plutinos, librating in the 2:3 mean-motion resonance with Neptune, and the cubewanos, a somewhat more distant population of nonlibrating objects with low orbital eccentricities. The existence of a 3:4 Neptune librator and a 3:5 Neptune librator was also suspected. These librators have now been confirmed, and a possible 4:7 librator and possible second 3:5 librator have also been found. The known and suspected multiple-opposition librators are listed in Table 1. Here it is important to note that the orbital semimajor axes $a$ (in $\mathrm{AU}$ ), eccentricities $e$ and inclinations $i$ (in degrees with respect to the 2000.0 ecliptic) are mean values that eliminate the large 12 -year and 30-year periodicities arising from the indirect perturbations by Jupiter and

Table 1. Librating Kuiper Belt objects.

\begin{tabular}{|c|c|c|c|c|c|c|c|c|}
\hline & & & $\bar{a}$ & $e$ & $i$ & $\bar{H}$ & Nep. & Ura. \\
\hline $3: 4$ & (36.41) & $1995 \mathrm{DA}_{2}$ & 36.39 & 0.073 & 6.5 & 8.0 & 8.0 & 14.5 \\
\hline \multirow[t]{15}{*}{$2: 3$} & (39.39) & 1993 RO & 39.30 & 0.199 & 3.7 & 8.0 & 12.5 & 11.4 \\
\hline & & $1995 \mathrm{YY}_{3} ?$ & 39.36 & 0.220 & 0.4 & 8.5 & 14.7 & 11.1 \\
\hline & & $1997 \mathrm{QJ}_{4}$ & 39.36 & 0.220 & 16.6 & 7.5 & 15.9 & 13.0 \\
\hline & & 1993 SB & 39.38 & 0.320 & 1.9 & 8.0 & 20.1 & 7.5 \\
\hline & & $1996 \mathrm{TP}_{66}$ & 39.42 & 0.330 & 5.7 & 6.5 & 22.0 & 6.9 \\
\hline & & $1994 \mathrm{JR}_{1}$ & 39.44 & 0.119 & 3.8 & 7.5 & 11.5 & 15.8 \\
\hline & & $1996 \mathrm{TQ}_{66}$ & 39.47 & 0.123 & 14.7 & 6.5 & 14.2 & 14.8 \\
\hline & & $1995 \mathrm{QZ}_{9}$ & 39.52 & 0.148 & 19.6 & 7.5 & 16.5 & 14.3 \\
\hline & & $1996 \mathrm{SZ}_{4}$ & 39.55 & 0.253 & 4.7 & 8.0 & 18.2 & 9.9 \\
\hline & & 1994 ТВ & 39.55 & 0.318 & 12.1 & 7.0 & 22.8 & 8.8 \\
\hline & & Pluto & 39.55 & 0.250 & 17.1 & -1.0 & & \\
\hline & & 1993 SC & 39.59 & 0.186 & 5.2 & 7.0 & 14.9 & 12.4 \\
\hline & & $1995 \mathrm{HM}_{5}$ & 39.60 & 0.253 & 4.8 & 8.0 & 15.2 & 10.6 \\
\hline & & $1996 R_{2}$ & 39.70 & 0.183 & 5.3 & 7.0 & 11.3 & 13.8 \\
\hline & & $1995 \mathrm{QY}_{9}$ & 39.76 & 0.265 & 4.8 & 7.5 & 11.7 & 9.2 \\
\hline \multirow[t]{2}{*}{$3: 5$} & $(42.25)$ & 1994 JS & 42.29 & 0.217 & 14.0 & 8.0 & 14.4 & 14.4 \\
\hline & & $1996 \mathrm{TR}_{66} ?$ & 42.35 & 0.219 & 12.4 & 7.5 & 16.9 & 14.4 \\
\hline $4: 7$ & $(43.65)$ & $1997 \mathrm{CV}_{29} ?$ & 43.67 & 0.183 & 8.0 & 7.0 & 11.8 & 16.5 \\
\hline
\end{tabular}


Table 2. Nonlibrating Kuiper Belt objects.

\begin{tabular}{|c|c|c|c|c|c|c|c|}
\hline & & & $a$ & $e$ & $i$ & $H$ & Nep. \\
\hline \multirow{3}{*}{$5: 8$} & $(41.12)$ & & & & & & \\
\hline & & $1997 \mathrm{RX}_{9} ?$ & 41.38 & 0.035 & 30.0 & 8.0 & 10.2 \\
\hline & & & 41.71 & 0.082 & 12.7 & 7.0 & 9.2 \\
\hline \multirow{5}{*}{$3: 5$} & $(42.25)$ & & & & & & \\
\hline & & $199 ?$ & 42.71 & 0.031 & 13.2 & 7.0 & 11.5 \\
\hline & & & 42.77 & 0.005 & 3.3 & 7.0 & 12.3 \\
\hline & & & 42.81 & 0.030 & 1.5 & 6.5 & 11.2 \\
\hline & & 199 & 42.94 & 0.041 & 1.7 & 7.0 & 11.3 \\
\hline \multirow[t]{3}{*}{$7: 12$} & (43.05) & & & & & & \\
\hline & & $1996 \mathrm{TO}_{66}$ & 43.47 & 0.131 & 27.4 & 4.5 & 10.2 \\
\hline & & $1997 \mathrm{C}$ & 43.53 & 0.029 & 1.5 & 6.5 & 12.0 \\
\hline \multirow{9}{*}{$4: 7$} & $(43.6$ & & & & 77 & 70 & 124 \\
\hline & & & 43.79 & 0.0 & 0.6 & 7.0 & 11.1 \\
\hline & & $29 ?$ & 43.84 & 0.025 & 1.0 & 5.0 & 12.5 \\
\hline & & & 43.92 & 0.124 & 7.3 & 6.0 & 9.2 \\
\hline & & & 43.94 & $0 . C$ & 2.2 & 5.0 & 13.4 \\
\hline & & 199 & 44.00 & 0.071 & 2.2 & 7.0 & 11.1 \\
\hline & & 199 & 44.04 & 0.109 & 31.7 & 7.0 & 9.6 \\
\hline & & 19 & 44.14 & 0.049 & 3.7 & 7.0 & 12.0 \\
\hline & & $1995 \mathrm{DC}_{2}$ & 44.15 & 0.068 & 2.3 & 7.0 & 11.0 \\
\hline $5: 9$ & $(44.4$ & & & & & & \\
\hline \multirow{3}{*}{$6: 11$} & 84 & & 44.11 & 0.081 & 2.9 & 6.5 & 10.9 \\
\hline & & & 45.09 & 0.0 & 19.1 & 6.5 & 12.7 \\
\hline & & $1996 \mathrm{~K}$ & 45.27 & 0.110 & 8.1 & 7.5 & 10.1 \\
\hline \multirow[t]{4}{*}{$7: 13$} & $(45.41)$ & & & & & & \\
\hline & & & & & 1. & 7.5 & 10.3 \\
\hline & & & 46.53 & 0.1 & 1.7 & 7.0 & 10.4 \\
\hline & & $1995 \mathrm{DB}_{2}$ & 46.62 & 0.140 & 4.1 & 7.5 & 10.1 \\
\hline & $(47.71)$ & & & & & & \\
\hline
\end{tabular}

Saturn on sun-centered orbits. The numbers in parentheses are the semimajor axes (in $\mathrm{AU}$ ) corresponding to the resonances. Following the absolute magnitude $H$, the entries "Nep." and "Ura." show the minimum distances (in AU) from Neptune and Uranus (the latter being of course quite small for the most eccentric 2:3 Neptune librators) within several millennia of the present time.

Table 2 lists the corresponding data (except for the minimum distances from Uranus) for the established cubewanos, which range over $41<a<47 \mathrm{AU}$, with $e$ rising to 0.14 for the most distant objects. The existence of several high- $i$ objects is an interesting new feature that warrants the need for searches at high ecliptic latitudes. The initial columns in this table show the positions of the resonances with 
Table 3. Centaurs and Scattered-Disk objects.

\begin{tabular}{lccrrrrrr}
\hline & $a$ & $e$ & $i$ & $H$ & Nep. & Ura. & Sat. & Jup. \\
\hline (2060) Chiron & 13.67 & 0.381 & 6.9 & 6.5 & 6.8 & 0.6 & 0.4 & 2.9 \\
(5145) Pholus & 20.30 & 0.572 & 24.7 & 7.0 & 0.3 & 4.9 & 0.6 & 3.5 \\
(7066) Nessus & 24.61 & 0.520 & 15.6 & 9.6 & 4.3 & 4.3 & 1.8 & 6.4 \\
(8405) 1995 GO & 18.05 & 0.620 & 17.6 & 9.0 & 6.4 & 0.8 & 0.2 & 2.1 \\
1994 TA & 16.79 & 0.303 & 5.4 & 11.5 & 7.9 & 1.4 & 5.0 & 6.4 \\
$1995 \mathrm{DW}_{2}$ & 25.06 & 0.247 & 4.1 & 8.0 & 1.0 & 0.2 & 8.6 & 12.8 \\
$1997 \mathrm{CU}_{26}$ & 15.78 & 0.170 & 23.4 & 6.5 & 12.7 & 2.8 & 4.4 & 8.1 \\
& & & & & & & & \\
$1996 \mathrm{TL}_{66}$ & 83.82 & 0.582 & 24.0 & 5.0 & 6.4 & & & \\
\hline
\end{tabular}

Neptune (out to sixth order) and possibly give a first indication of the presence of "Kirkwood gaps"- except, of course, for the high-e 3:5 and suspected 4:7 librators shown in Table 1. It is also noteworthy that the population seems to terminate inside the 1:2 resonance, at least for objects of the size represented by the observed $H$ values, although this requires further investigation. Sensitivity of the detection probability to distance, and hence to $a$, suggests that the observed plutino-cubewano ratio, 0.65 , is about twice what it would be for objects down to a given minimum size.

One of the objects discovered in late 1996 at the general distance of the Kuiper Belt was the perihelic detection (Luu et al. 1997) of the single known member, 1996 $\mathrm{TL}_{66}$, of what has been termed the "scattered disk", a presumably large population of objects in orbits of rather large eccentricity and moderate inclination that at perihelion can have modest interactions with Neptune. The scattered-disk objects have an obvious dynamical symmetry with the centaurs that exist in rather unstable orbits within the realm of the giant planets and that in some cases interact with Neptune at aphelion. Table 3 gives orbital data for the seven known centaurs (three of which are intrinsically fainter than any of the entries in Tables 1 and 2) and the one known scattered-disk object. For the centaurs, columns 'Sat.' and 'Jup.' also show the minimum distances (again within just millennia of the present) from Saturn and Jupiter. The physical similarity of at least some of the centaurs to the short-period comets has been suspected (Kowal et al. 1979) or known (Meech and Belton 1990) for some time, and dynamical analyses (Duncan et al. 1995) strongly suggest that the centaurs are objects in transition between the Kuiper Belt (where they presumably originated) and the Jupiter Family of comets with their aphelia generally in the vicinity of Jupiter.

The objects tabulated in this paper have all been observed at more than one opposition. In addition, there are 26 single-opposition Kuiper Belt candidates, at least half of which are lost. 


\section{References}

Duncan, M. J., Levison, H. F. and Budd, S. M.:1995, Astron. J, 110, 3073-3081.

Kowal, C. T., Liller, W. and Marsden, B. G: 1979, In Dynamics of the Solar System, ed. R. L. Duncombe, IAU Symp. No. 81, 245-250.

Luu, J., Marsden, B. G., Jewitt, D., Trujillo, C. A., Hergenrother, C. W., Chen, J. and Offutt, W. B.: 1997, Nature, 387, 573-575.

Marsden, B. G.: 1996a, Searching for comets and planets. In Completing the Inventory of the Solar System, ed. T. E. Rettig and J. M. Hahn, ASP Conf. Ser, 107, 193-207.

Marsden, B. G.: 1996b, From telescope to MPC: organizing the minor planets. In Ephemerides and Astrometry of the Solar System, ed. S. Ferraz-Mello, B. Morando and J.-E. Arlot, IAU Symp. No. $172,153-164$.

Marsden, B. G.:1998, Kuiper Belt: securing adequate orbital data. In Asteroids, Comets, Meteors 1996, ed. A. C. Levasseur-Regourd and M. Fulchignoni, COSPAR, in press.

Meech, K. J. and Belton, M. J. S.: 1990, Astron. J, 100, 1323-1338. 